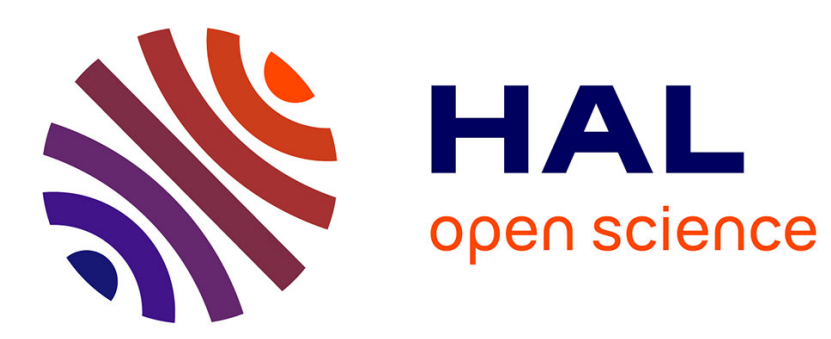

\title{
Arterial Bandwidth Maximization via Signal Offsets and Variable Speed Limits Control
}

Giovanni de Nunzio, Gabriel Gomes, Carlos Canudas de Wit, Roberto Horowitz, Philippe Moulin

\section{- To cite this version:}

Giovanni de Nunzio, Gabriel Gomes, Carlos Canudas de Wit, Roberto Horowitz, Philippe Moulin. Arterial Bandwidth Maximization via Signal Offsets and Variable Speed Limits Control. CDC 2015 54th IEEE Conference on Decision and Control, Dec 2015, Osaka, Japan. hal-01197443

HAL Id: hal-01197443

https://hal.inria.fr/hal-01197443

Submitted on 11 Sep 2015

HAL is a multi-disciplinary open access archive for the deposit and dissemination of scientific research documents, whether they are published or not. The documents may come from teaching and research institutions in France or abroad, or from public or private research centers.
L'archive ouverte pluridisciplinaire $\mathbf{H A L}$, est destinée au dépôt et à la diffusion de documents scientifiques de niveau recherche, publiés ou non, émanant des établissements d'enseignement et de recherche français ou étrangers, des laboratoires publics ou privés. 


\title{
Arterial Bandwidth Maximization via Signal Offsets and Variable Speed Limits Control
}

\author{
Giovanni De Nunzio, Gabriel Gomes, Carlos Canudas de Wit, Roberto Horowitz, Philippe Moulin
}

\begin{abstract}
The problem of maximizing bandwidth along an arterial is here addressed by use of two combined control actions: traffic lights offsets and variable speed limits. The optimization problem has been enriched in order to account for traffic energy consumption and network travel time, thus avoiding impractical or undesirable solutions. A traffic microscopic simulator has been used to assess the performance of the proposed technique in terms of energy consumption, travel time, idling time, and number of stops. The theoretical bandwidth proves to be well correlated with idling time and number of stops, while the variable speed limits control shows interesting advantages in terms of energy consumption without penalizing the travel time. An analysis of the Pareto optimum has been carried out to help the designer choose a trade-off in the multiobjective optimization.
\end{abstract}

Index Terms - bandwidth maximization, energy consumption, signal coordination, variable speed limits

\section{INTRODUCTION}

Benefits of traffic lights coordination on traffic relief is undeniable. Well coordinated traffic lights can reduce travel time, delay, and unnecessary stops. As a consequence, they improve mobility and access, reduce driver frustration, and energy and fuel consumption. Potential beneficial effects may be observed also in reduced rerouting through nearby neighborhoods, pollution, and fewer accidents.

Many traffic-signal optimization algorithms, both fixedtime (e.g. TRANSYT) and traffic-responsive (e.g. SCOOT, SCATS), have found wide practical use. The objective of these traffic-management strategies is to reduce the total length of the queues in the monitored network area, and consequently reduce delays. Though delay reduction and minimization of stops are considered as important performance targets, maximization of progression bands is still considered essential by many practitioners. For this reason, some later versions of the cited algorithms (e.g. TRANSYT$7 \mathrm{~F})$ also incorporate progression measures.

The size of the progression band enabled by a sequence of signalized intersections is known as the bandwidth. Maximizing the bandwidth of a signalized arterial corresponds to maximizing the time during which vehicles can drive the

G. De Nunzio, Ph.D. Candidate at IFPen, Rueil-Malmaison, France, giovanni.de-nunzio@ifpen.fr

G. Gomes, Research Engineer, California PATH, UC Berkeley, USA, gomes@path.berkeley.edu

C. Canudas de Wit, Director of Research at CNRS, GIPSA-Lab, NeCS team, Grenoble, France, carlos.canudas-de-wit@gipsa-lab.fr

R. Horowitz, Professor at Department of Mechanical Engineering, Director of California PATH, UC Berkeley, USA, horowitz@berkeley.edu

P. Moulin, Department of Control Signal and Systems at IFPen, RueilMalmaison, France, philippe.moulin@ifpen.fr entire length of the arterial without stopping. Bandwidthbased signal timings are generally preferred because they meet driver expectations about traffic fluidity [1].

The first mathematical formulation of the bandwidth maximization problem was given by Morgan and Little in [2]. The nature of the problem was combinatorial and it addressed a simplified two-stage version, thus neglecting more complex phases. An extension of this first work was published by Little in [3], where the problem was formulated for the first time as a mixed-integer linear program (MILP).

This pioneering work evolved into MAXBAND [4], which offered as main features the possibility to choose cycle times and offsets to maximize bandwidth in the two directions of travel, as well as the possibility to deal with triangular networks and more complex turning phases at the intersections. MAXBAND gave rise to several research efforts and extensions, first in order to solve triangular networks (MAXBAND-86) [5], and then to allow different bandwidths for each segment of a single arterial (MULTIBAND) [6] or a network of arterials (MULTIBAND-96) [7], [8]. Recent research has combined link-based and arterial-based techniques [9], as well as introduced network partitioning algorithms in order to deal with larger networks [1]. The undersaturated traffic assumption was relaxed in [10], [11].

All of the mentioned works and software packages for bandwidth maximization are based on the original MILP formulation in [3]. However the results in [12] show that the arterial bandwidth maximization problem can also be cast as a linear program (LP) without the need for integer variables. This result is based on the observation that the integer unknowns are closely related to the inter-signal travel times, and can therefore be computed a priori.

In the original formulation of the arterial bandwidth maximization by Little [3] and the MAXBAND software package [4], the speed in the segments of the two-way arterial was allowed to vary as an additional decision variable of the MILP to further improve the progression bands. However both in the software implementation and testing of MAXBAND, the potential benefits induced by the variable speed limits on actual traffic performance were never assessed.

A general and often misleading assumption made by drivers is that an increase in speed will lead to a proportionate decrease in travel time. However, travel time is more dependent on congestion and roadway design and geometry factors than on the posted speed limits. [13] has pointed out that at traffic density levels where a flow can be maintained, it is theoretically possible that lower speed limits could actually bring about a reduction in overall travel time. We will also 
show in this paper that a lower average speed limit may translate into a similar or lower average travel time, while drastically reducing the environmental impact.

In this work, the two-way arterial bandwidth maximization problem is addressed with a particular focus on the benefits induced by the variable speed limits, and on the energy consumption reduction. The present mathematical formulation, inspired by the idea in [12], allows to solve the one-way bandwidth maximization problem as an LP, even with segment speeds as decision variables. However, as we will see, the two-way problem with internal offsets constraints requires the formulation of the problem as an MILP. The first contribution of the paper lies in the addition of terms representing traffic energy consumption and network travel time to the objective function of the two-way arterial bandwidth maximization. The variable speed limits allow to reach higher theoretical bandwidths but might induce driving discomfort and higher energy consumption if the variability of the speeds between the segments is too high. Furthermore, optimal solutions with low speed limits are to be avoided, in trade-off with the energy consumption. The second contribution of the paper is given by the extensive evaluation of the benefits of bandwidth maximization via a microscopic traffic simulator. Bandwidth is a theoretical quantity and a correlation with known traffic performance metrics needs to be established in order to justify its use. An analysis of the Pareto optimality will allow for an efficient design of the optimization function. The combined control of offsets and speed limits turns out to have a large impact on energy consumption with no degradation of the travel time.

The paper is organized as follows. Section II describes the problem and the notation. Section III contains the optimization problem formulation. The simulation setup is presented in Section IV. Results of the bandwidth optimization and traffic performance are shown in Section V. Conclusions are given in Section VI.

\section{Problem Setup}

A two-way arterial with $n$ signalized intersections is considered. The two opposing directions of travel are referred to as inbound and outbound. Intersections are located at $x_{1}<x_{2}<\ldots<x_{n}$, with the subscript index increasing in the outbound direction. All quantities related to the inbound direction are denoted with an overbar. The travel speeds in the $n-1$ segments are assumed to be equal to the imposed speed limits, and denoted as $v_{i}$ and $\bar{v}_{i}$.

The outbound and inbound travel times on segment $i$ are,

$$
\begin{aligned}
& t_{i}=\frac{x_{i+1}-x_{i}}{v_{i}}>0, \quad i \in\{1, \ldots, n-1\} \\
& \bar{t}_{i}=\frac{\bar{x}_{i}-\bar{x}_{i+1}}{\bar{v}_{i}}<0, \quad i \in\{1, \ldots, n-1\}
\end{aligned}
$$

with the segment length being $L_{i}=x_{i+1}-x_{i}$.

The $n$ signals are to be coordinated with a common cycle time $C$, which is assumed to be given and fixed. All signals are also on a fixed schedule, and denote with $g_{i}\left(\bar{g}_{i}\right)$ the green times at intersection $i$ in the outbound (inbound) direction.

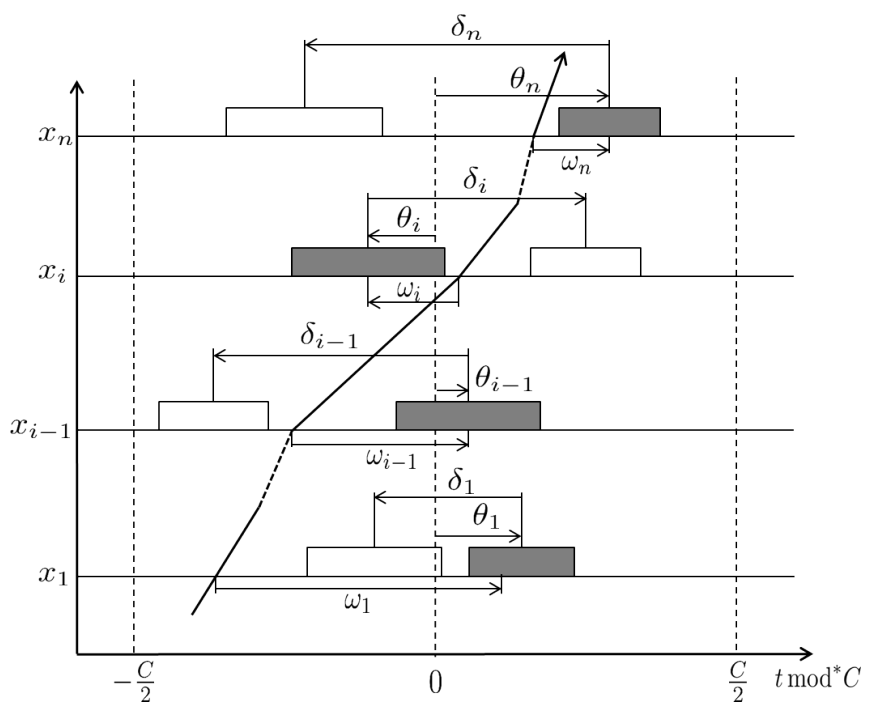

Fig. 1: Green phases of the outbound direction of travel are shaded. Green phases in the inbound direction are in white. The travel speed trajectory and the basic notation are indicated only for the outbound direction for clarity.

The mapping of time from the real domain onto the interval $[-C / 2, C / 2]$ is performed with a non-standard modulo operator which will be denoted $\bmod ^{*}$ [12]. $p \bmod ^{*} C$ returns the distance from $p$ to the nearest integer multiple of $C$, with negative sign if the integer is to the right of $p$ and positive sign if it is to the left of $p$. The absolute offsets $\theta_{i}\left(\bar{\theta}_{i}\right)$ are defined as the displacement of the center of the green phase of the outbound (inbound) direction at intersection $i$ with respect to a fixed coordinate system (see Figure 1). The absolute offsets are in $[-C / 2, C / 2]$. The internal offset, or the relative displacement of the centers of the inbound and outbound green phases, is defined as:

$$
\delta_{i}=\left(\bar{\theta}_{i}-\theta_{i}\right) \bmod ^{*} C
$$

In addition to the absolute offsets, the relative offsets $\omega_{i}$ and $\bar{\omega}_{i}$ are defined. These are measured with respect to the outbound and inbound moving coordinate frames, which travel at speed $v_{i}$ and $\bar{v}_{i}$ respectively. The outbound relative offset $\omega_{i}$ is defined as the time between the passage of the outbound moving coordinate frame and the center of the nearest outbound green phase. A corresponding definition applies to the inbound relative offset. The relative offsets are also in $[-C / 2, C / 2]$. Note that the moving frame does not stop at the red signals.

The conversion between absolute and relative offsets may be derived as follows:

$$
\left(\omega_{i-1}-\theta_{i-1}+\theta_{i}-\omega_{i}-t_{i-1}\right) \bmod ^{*} C=0
$$

Therefore the recursive formula for relative offsets is:

$$
\omega_{i}=\left(\omega_{i-1}-\theta_{i-1}+\theta_{i}-t_{i-1}\right) \bmod ^{*} C
$$

which eventually yields:

$$
\omega_{i}=\left(\omega_{1}-\theta_{1}+\theta_{i}-\sum_{k=1}^{i-1} t_{k}\right) \bmod ^{*} C
$$


The inverse formula allows to determine the absolute offsets from the relative offsets:

$$
\theta_{i}=\left(\theta_{1}-\omega_{1}+\omega_{i}+\sum_{k=1}^{i-1} t_{k}\right) \bmod ^{*} C
$$

These formulas apply with overbars for the inbound direction. Because the map between relative and absolute offsets is invertible, we are free to formulate the bandwidth maximization problem in terms of either set of unknowns.

Bandwidth is defined as the time interval in which vehicles can travel the length of the arterial without stopping.

\section{PROBLEM FORMULATION}

The objective of this work is to solve the two-way bandwidth maximization problem, using both offset control and variable speed limits (VSL) control. The degrees of freedom of the optimization problem will be the relative offsets $\omega_{i}$ and $\bar{\omega}_{i}$, together with the travel times $t_{i}$ and $\bar{t}_{i}$.

The two-way problem has been formulated in [12] as a continuous linear program, without use of integer variables, exploiting the a priori information about the speeds in each segment of the arterial network. Only the offsets were used as decision variables. This work extends the previous result by introducing the speeds in the different segments of the network as additional decision variables. It will be shown that a higher bandwidth is achieved thanks to VSL control. However, the objective of the current analysis is also to avoid impractical solutions, as well as to evaluate the actual benefits of the maximization of the theoretical bandwidth on traffic performance. In particular, the optimization will account for traffic energy consumption, and travel time.

In order to express both outbound and inbound bandwidth (i.e. $b$ and $\bar{b}$ ) in terms of the relative offsets, the green phases are translated along the outbound and inbound coordinate frames to $x=x_{1}$. This operation induces a mapping from $\delta_{i}$ to $\delta_{i}^{0}$ with $i \in\{1, \ldots, n\}$. The mapping is given by (see [12] for details):

$$
\delta_{i}^{0}=\left(\delta_{i}+\sum_{k=1}^{i-1}\left(t_{k}-\bar{t}_{k}\right)\right) \bmod ^{*} C
$$

with $\delta_{i}^{0} \in[-C / 2, C / 2]$. Note that the $\delta_{i}$ are given. The equality constraint that fixes the internal offset is given by:

$$
\omega_{i}-\bar{\omega}_{i}=\delta_{1}^{0}-\delta_{i}^{0}
$$

In vector notation:

$$
\bar{\omega}=\omega-\delta
$$

with $\omega \triangleq\left(\omega_{1}, \ldots, \omega_{n}\right)$ and $\delta \triangleq\left(\delta_{1}^{0}, \delta_{1}^{0}-\delta_{2}^{0}, \ldots, \delta_{1}^{0}-\delta_{n}^{0}\right)$.

\section{Directional interference constraints}

The progression band, in the case of fixed green times and no platoon dispersion, is equivalent to the intersection of all the green intervals, measured with respect to the moving coordinate frame. Hence, as demonstrated in [12], the bandwidth is the size of the intersection of the following set of intervals:

$$
\left\{\left[\omega_{i}-g_{i} / 2, \omega_{i}+g_{i} / 2\right]: i \in\{1, \ldots, n\}\right\}
$$

This leads to the expression for the outbound bandwidth:

$$
b\left(\omega_{1}, \ldots, \omega_{n}\right)=\max \left(0, \min _{i, j \in\{1, \ldots, n\}}\left(\omega_{i}-\omega_{j}+g_{i, j}\right)\right)
$$

where $g_{i, j} \triangleq\left(g_{i}+g_{j}\right) / 2$. Equation (11) is the solution to the following linear program:

$$
\begin{array}{ll}
\max & b \\
\text { s.t. } & b \leq g_{i, j}+\omega_{i}-\omega_{j} \\
& b \geq 0
\end{array}
$$

Analogous linear program applies to the inbound bandwidth.

By combining the two problems, and imposing the constraint (10), the constraints can be written as:

$$
\begin{array}{ll}
b \leq g_{i, j}+\omega_{i}-\omega_{j}, & \forall i \neq j \in\{1, \ldots, n\} \\
\bar{b} \leq \bar{g}_{i, j}+\omega_{i}-\omega_{j}+\delta_{i}^{0}-\delta_{j}^{0}, & \forall i \neq j \in\{1, \ldots, n\}
\end{array}
$$

and in matrix form:

$$
\begin{aligned}
& b \mathbf{1} \leq \Omega \cdot \omega+\frac{1}{2}|\Omega| \cdot \mathbf{g} \\
& \bar{b} \mathbf{1} \leq \Omega \cdot \omega+\Delta \cdot \delta^{\mathbf{0}}+\frac{1}{2}|\Omega| \cdot \overline{\mathbf{g}}
\end{aligned}
$$

where $\Omega, \Delta \in \mathbb{R}^{n(n-1) \times n}$. The upper and lower bounds for the bandwidths are:

$$
\begin{array}{ll}
b \leq g^{*}, & b \geq 0 \\
\bar{b} \leq \bar{g}^{*}, & \bar{b} \geq 0
\end{array}
$$

where $g^{*}=\min \left(g_{i}\right)$ and $\bar{g}^{*}=\min \left(\bar{g}_{i}\right)$. We have therefore a total of $[2 n(n-1)+4]$ constraints for the bandwidths.

\section{Travel time constraints}

The travel times in the two directions $t_{i}$ and $\bar{t}_{i}$ are now decision variables of the optimization problems. The maximum and minimum speed limits are assumed to be the same in both directions, and therefore the bounds of the travel times will depend on the length of the segments. These constraints can be written in vector form as follows:

$$
\begin{aligned}
\mathbf{t}_{\min } & \leq \mathbf{t} \leq \mathbf{t}_{\max } \\
-\mathbf{t}_{\max } & \leq \overline{\mathbf{t}} \leq-\mathbf{t}_{\text {min }}
\end{aligned}
$$

where the vector of travel times is $\mathbf{t}=\left(t_{1}, \ldots t_{n-1}\right)$, and the bounds on the travel times are:

$$
t_{i, \min }=\frac{L_{i}}{v_{\max }}, \quad t_{i, \max }=\frac{L_{i}}{v_{\min }}
$$

Analogous equations apply to the inbound direction. This defines $4(n-1)$ constraints.

\section{Internal offset constraints}

In this framework, the displaced internal offsets $\delta_{i}^{0}$ are not known a priori, but depend on decision variables $t_{i}$ and $\bar{t}_{i}$ through (8). The difficulty is represented by the modulo operator, which has to be accounted for in the optimization.

Constraints with modulo operator break the standard continuous form of the problem, which now becomes an integer programming problem. Constraint (8) can be rewritten as:

$$
\delta_{i}+\sum_{k=1}^{i-1}\left(t_{k}-\bar{t}_{k}\right)=\alpha_{i} C+\delta_{i}^{0}
$$


where $\alpha_{i} \in \mathbb{N}$, with $i \in\{2, \ldots, n\}$, is a new integer decision variable. Note that the $\delta_{i}$ are given, $\delta_{1}^{0}=\delta_{1}$ and therefore $\alpha_{1}=0$. In matrix form, bringing the unknowns on the lefthand side, the $n$ constraints on $\delta^{0}$ are as follows:

$$
\delta^{\mathbf{0}}-\Psi \cdot \mathbf{t}+\Psi \cdot \overline{\mathbf{t}}+\alpha \cdot C=\delta
$$

where the matrix $\Psi \in \mathbb{R}^{n \times(n-1)}$, and the vector of integer variables is $\alpha=\left[0, \alpha_{2}, \ldots, \alpha_{n}\right]^{T}$.

\section{Optimization Problem}

The objective is to maximize the sum of the bandwidth in the two directions. The problem will be solved with respect to the outbound relative offsets and the transformed internal offsets (i.e. offset control), the travel times (i.e. variable speed limits), and the integer variables that keep count of the number of cycles. Therefore the number of unknowns of the optimization problem is $5 n-1$ :

$$
\begin{aligned}
\mathbf{x}= & {\left[b, \bar{b}, \omega_{1}, \ldots, \omega_{n}, \delta_{1}^{0}, \ldots, \delta_{n}^{0}, t_{1}, \ldots, t_{n-1},\right.} \\
& \left.\bar{t}_{1}, \ldots, \bar{t}_{n-1}, \alpha_{2}, \ldots, \alpha_{n}\right]^{T}
\end{aligned}
$$

Besides bandwidth maximization, it is also of interest to minimize the variance of the imposed speed limits, as well as the travel time. The former may be thought of as a control variance term, or as a comfort term because the drivers would be less willing to follow a highly variable speed advisory. This term also serves to reduce vehicle accelerations that increase energy consumption. The second term drives the optimization away from trivial solutions with impractically low velocities. These additional terms may be written as a function of $\mathbf{x}$.

Smoothness term: By discouraging large variations in speed between segments, the energy consumption associated with the VSL is minimized. The quantity to be minimized for the outbound direction is:

$$
\left\|\frac{L_{i}}{t_{i}}-\frac{L_{i+1}}{t_{i+1}}\right\|_{1}, \quad i \in\{1, \ldots, n-2\}
$$

The same expression applies for the inbound direction, with $\bar{t}_{i}$ and $\bar{t}_{i+1}$. In order to avoid the introduction of nonlinear terms in the objective function, we will approximate (24) with a linear function corresponding to its numerator. Therefore the smoothness term can be rewritten as:

$$
\left\|L_{i} t_{i+1}-L_{i+1} t_{i}\right\|_{1}, \quad i \in\{1, \ldots, n-2\}
$$

which can be expressed in matrix form with:

$$
\left\|\mathcal{L}^{*} \cdot \mathbf{t}\right\|_{1}
$$

where $\mathcal{L}^{*} \in \mathbb{R}^{(n-2) \times(n-1)}$. Analogously for the inbound direction. This simplification does not affect the convexity of the problem and in particular does not modify the minimum of the smoothness term, which will still be reached when the speeds are equal. The approximation will change the shape of the function away from the minimum, but its convexity will still drive the optimization towards the desired point. In this way, the decision variables $t$ and $\bar{t}$ do not appear at the denominator and the linearity of the smoothness term is preserved.
Travel Time term: In order to minimize the theoretical travel time induced by the speed limits, the $L_{1}$-norm of $t$ is also to be minimized. Therefore the travel time term for the outbound direction in vector form may be written as:

$$
\|\mathbf{t}\|_{1}
$$

Analogously for the inbound direction.

Final Formulation: The problem can be finally formulated as a nonlinear integer program as follows:

$$
\left\{\begin{array}{cl}
\max _{\mathbf{x}} & b+\bar{b}-\lambda_{1}\left(\left\|\mathcal{L}^{*} \mathbf{t}\right\|_{1}+\| \mathcal{L}^{*} \overline{\mathbf{t}}||_{1}\right)-\lambda_{2}\left(\|\mathbf{t}\|_{1}+\|\overline{\mathbf{t}}\|_{1}\right) \\
\text { s.t. } & b \mathbf{1} \leq \Omega \cdot \omega+\frac{1}{2}|\Omega| \cdot \mathbf{g} \\
& \bar{b} \mathbf{1} \leq \Omega \cdot \omega+\Delta \cdot \delta^{\mathbf{0}}+\frac{1}{2}|\Omega| \cdot \overline{\mathbf{g}} \\
& b \leq g^{*} \\
& b \geq 0 \\
& \bar{b} \leq \bar{g}^{*} \\
& \bar{b} \geq 0 \\
& \mathbf{t}_{\min } \leq \mathbf{t} \leq \mathbf{t}_{\max } \\
& -\mathbf{t}_{\max } \leq \overline{\mathbf{t}} \leq-\mathbf{t}_{\min } \\
& \delta^{\mathbf{0}}-\Psi \cdot \mathbf{t}+\Psi \cdot \overline{\mathbf{t}}+\alpha \cdot C=\delta
\end{array}\right.
$$

with $\lambda_{1}$ and $\lambda_{2}$ being the optimization weights.

The objective function can be linearized by introducing slack variables that behave as maximum bounds for the L1norms [14]. Four such variables $\gamma \in \mathbb{R}^{(n-2)}, \bar{\gamma} \in \mathbb{R}^{(n-2)}$, $\tau \in \mathbb{R}^{(n-1)}$ and $\bar{\tau} \in \mathbb{R}^{(n-1)}$ are introduced such that:

$$
\begin{aligned}
& \left|\mathcal{L}^{*} \cdot \mathbf{t}\right| \leq \gamma \quad \text { and } \quad|\mathbf{t}| \leq \tau \\
& \left|\mathcal{L}^{*} \cdot \overline{\mathbf{t}}\right| \leq \bar{\gamma} \quad \text { and } \quad|\overline{\mathbf{t}}| \leq \bar{\tau}
\end{aligned}
$$

Thus, the vector of the unknowns is extended:

$$
\mathbf{z}=[\mathbf{x}, \gamma, \bar{\gamma}, \tau, \bar{\tau}]^{T}
$$

and new constraints appear in the optimization problem.

Therefore the problem can be finally formulated as a MILP with $(9 n-7)$ unknowns and $\left(4 n^{2}+11 n-11\right)$ constraints.

\section{Simulation SetuP}

The goal of the simulation campaign is to assess the performance of our optimization problem with respect to the one solved in [12]. Specifically, the proposed approach, by means of both offset and variable speed limits control, is expected to achieve a higher bandwidth than the offsetsbased optimization. Furthermore, the benefits analysis will not be conducted only at the theoretical level by comparing the levels of total bandwidth achieved. Also real traffic performance metrics, such as energy consumption, travel time, idling time at the traffic lights and number of stops, will be analyzed.

\section{Numerical Implementation}

The two-way bandwidth optimization problem (28) has been implemented in Matlab, using the native solver intlinprog for MILPs. For the sake of comparison, the 
problem has been always solved along with the one formulated in [12], in order to directly observe the differences between the two formulations and the benefits introduced by VSL in terms of theoretical bandwidth.

The computation time is negligible: 0.3 seconds for the problem here presented, 0.15 seconds for the problem presented in [12], for an arterial network with 10 intersections. It has also been observed that the MILP here presented scales better with the increasing number of intersections, whereas the simpler offsets-based optimization runs more slowly when $n$ increases. For instance, for $n=20$, the MILP optimization converges in about 0.4 seconds, while the LP converges in about 0.9 seconds. This can be explained by the generally larger space of optimal solutions available when combining offset and VSL control. In other words, as $n$ increases, the offset-only optimization search for the solution in a smaller and smaller space, therefore taking longer to converge. On the contrary, the combined optimization converges faster to one possible optimal solution because the search space is larger. Based on this, it is also possible to conclude that the integer variables in the optimization problem under analysis do not affect significantly the convergence time.

The simulations were run on a laptop with an Intel(R) Core(TM) i7-2760QM at $2.40 \mathrm{GHz}$ and $8 \mathrm{~GB}$ of RAM.

\section{Microscopic Traffic Simulator}

Aimsun was adopted as microscopic simulator for the tests. The traffic demand used in the traffic simulation was required to be feasible, in the sense that the green times were sufficient to dissipate the queues in each cycle. A conservative upper bound for the feasible demand is:

$$
D \leq D_{\max }=\frac{g^{*}}{C} \varphi_{m}\left(v_{\min }\right)
$$

where $g^{*}$ is the minimum of the green times in the signalized corridor, and $\varphi_{m}\left(v_{\min }\right)$ is the demand/supply saturation function for the imposed minimum speed limit [15]. This applies to both directions of travel.

A communication protocol between Aimsun and Matlab was set up in order to retrieve from the microscopic simulator information about the vehicles in the network, at each simulation step. Namely the vehicles send information about their position, current speed and acceleration. These data are used to compute traffic performance metrics. Each one of the performance metrics was obtained per segment and per vehicle completing the trip on the segment. Vehicles that at the end of the simulation time horizon remain in the network, do not fully contribute to the overall performance. After computing the performance per segment and per vehicle, the average performance per segment was obtained. In the following analysis, only the network-wide performance is reported, that is the sum over the two directions of travel of the mean performance in each segment. The performance metrics used in this work are defined as follows:

Travel time: The time a vehicle spends in the network.

Idling time: The time a vehicle spends at zero speed.

Number of Stops: The number of non-consecutive idling periods of a vehicle in the network.
Energy consumption: The energy is defined as the integration over time (i.e. the time the vehicle is in the segment) of the power demand.

$$
E=\int_{0}^{T_{f}} P \mathrm{~d} t=\int_{0}^{T_{f}} b_{1} u v \mathrm{~d} t
$$

The torque $u$ is derived from the vehicle longitudinal model of the vehicle [16]:

$$
\begin{aligned}
& \dot{x}=v \\
& \dot{v}=h_{1} u-h_{2} v^{2}-h_{3} v-h_{0}
\end{aligned}
$$

where $u$ is a function of the vehicle speed and acceleration. The constants $b_{i}$ and $h_{i}$ are obtained as in [17].

\section{EXPERIMENTS}

\section{Bandwidth degradation}

The first experiment aims at showing the degradation of the theoretical bandwidth with the increasing number of signalized intersections on the arterial. Several network parameters have been varied randomly in order to test different sets of green times, segment lengths, and internal offsets. The range of network parameters used in the stochastic simulations is shown in Table I.

TABLE I: Network parameters range

\begin{tabular}{lll}
\hline $\mathrm{n}$ & {$[3, \ldots, 15]$} & number of intersections (per direction) \\
$\mathrm{C}$ & 60 & cycle time $[\mathrm{s}]$ \\
$\mathrm{g}$ & {$[0.4,0.6]$} & green signal split ratio \\
$\mathrm{L}$ & {$[225,375]$} & segment lengths $[\mathrm{m}]$ \\
$\delta$ & {$[-C / 2, C / 2]$} & internal offset $[\mathrm{s}]$ \\
{$\left[v_{\min }, v_{\max }\right]$} & {$[15,50]$} & speed limits $[\mathrm{km} / \mathrm{h}]$ \\
\hline
\end{tabular}

In order to extensively explore the space of varying parameters, for each number of intersections $n$, the other parameters (i.e. green split ratio, segment length, and internal offset) were allowed to vary randomly within the specified ranges. A total of 10,000 simulations per each value of $n$ were run (i.e. 130,000 total different configurations). Furthermore, for each randomly generated configuration, both problem (28) and the offset-based bandwidth maximization presented in [12] were solved. The comparison of the theoretical bandwidth achieved by the two optimizations is shown in Figure 2.

In the graph, the solid lines represent the average bandwidth varying with the number of intersections. The vertical error bars at each value of $n$ represent the standard deviation of the bandwidth calculated over the random set of parameters. As $n$ increases the bandwidth decreases for both types of control action. However control of both offsets and VSL results to be significantly more effective, especially for a lower number of intersections. Note that for each $n$, the bandwidth achieved using the proposed approach is always greater than the one obtained using only offset control.

The weights used in the optimization are $\lambda_{1}=\lambda_{2}=0.5$. Interestingly, the red line would move with the selection of 


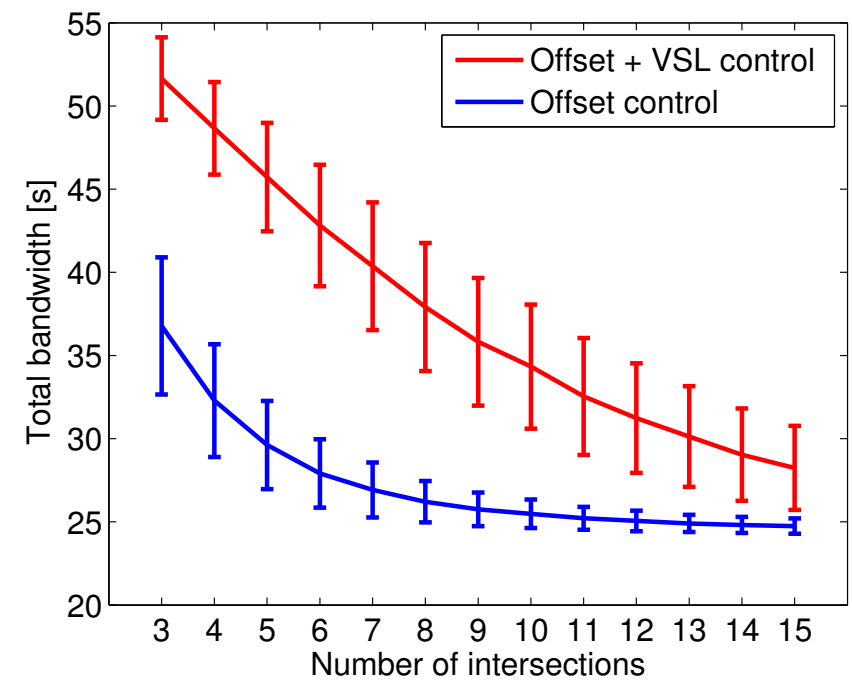

Fig. 2: Bandwidth degradation with number of intersections.

weights $\lambda_{1}$ and $\lambda_{2}$. Specifically, when $\lambda_{2}>>0$, the red line would coincide with the blue one, meaning that the presented optimization is forced to select the maximum speeds. When the two weights are set to zero, the red line would move up at its maximum, meaning that the VSL would be fully employed to maximize only the total bandwidth.

\section{Microscopic simulation}

For the microscopic simulations a single random network configuration was selected. The network parameters utilized in Aimsun are summarized in Table II.

TABLE II: Network parameters for microscopic simulation

\begin{tabular}{lll}
\hline$n$ & 6 & number of intersections \\
$g$ & {$[33,30,25,28,31,26]$} & outbound green times [s] \\
$\bar{g}$ & {$[33,27,35,27,33,26]$} & inbound green times [s] \\
$L$ & {$[268.1,238.7,311.4,327.5,307]$} & length of the segments [m] \\
$\delta$ & {$[25,-21,-21,-19,-22,-3]$} & internal offsets [s] \\
$D$ & 500 & traffic demand [veh/h] \\
\hline
\end{tabular}

For the choice of macroscopic network parameters (i.e. jam density, congestion speed, etc.), the feasible demand in (30) is $D_{\max }=536 \mathrm{veh} / \mathrm{h}$; therefore the simulations were conducted using a traffic demand close to the limit of existence of a green wave.

Given these network parameters, the two bandwidth optimization problems were solved, in order to obtain the control parameters to be tested in the microscopic simulator.

For the performance assessment of the combined optimization of offsets and speed limits, the space of the weights $\lambda_{1}$ and $\lambda_{2}$ of the multi-objective function has been spanned. The results are reported in Fig. 3.

In Fig. 3 it is evident how the theoretical bandwidth is monotonically decreasing in the direction of the increasing $\lambda_{1}$ and $\lambda_{2}$. Higher weights on the smoothness term and/or the travel time term in the optimization would achieve smoother speed limits profiles between adjacent systems and/or higher overall speed limits. Therefore, for high values of $\lambda_{2}$ the optimization converges towards the blue area of the bandwidth plot, which is equivalent to the offset-only optimization problem. In fact the theoretical bandwidth achieved is at its minimum, and equal to the one achieved by the offset control and reported in Table III. The benefits of the VSL for bandwidth maximization are evident for lower values of the weights $\lambda_{1}$ and $\lambda_{2}$.

In Fig. 3 also the energy consumption and the travel time computed from the microsimulation data are reported. It is interesting to observe how the traffic performance metrics are correlated with the theoretical bandwidth. In particular energy consumption is low in the area of maximum bandwidth, since higher bandwidth reduces also the idling time and the number of stops. However, the operation area of minimum energy consumption grows for low values of $\lambda_{2}$ and high values of $\lambda_{1}$, showing that lower and smoother speed limits benefit the energy consumption. Interestingly also travel time is minimized in the area of higher bandwidth, even though the average total speed limit is higher in the right-most side of the contour plots. This is an interesting demonstration of the fact that higher speed limits do not always correspond to lower travel times. For high $\lambda_{1}$ and small $\lambda_{2}$ the travel time increases because the optimization forces the speed limits to be as close as possible, without giving priority to high speeds. Furthermore, giving priority to smoothness or high speed limits causes the system to drift away from the high bandwidth zone. Where the bandwidth is minimum, the performance metrics worsen significantly.

A Pareto efficiency analysis has been conducted on the problem in order to assist the designer in making the choice of weights in the objective function. The Pareto frontier is defined as the set of points that cannot be said to be strictly worse than any other belonging to the set. All the points on the frontier are equivalently Pareto-efficient. In order to find the set of Pareto-efficient points, a standard non-dominated sorting algorithm was used. The adopted methodology is described as follows. The entire set of solutions obtained from the optimization problem (28) for the three objectives and for different weights combinations is searched for nondominated solutions. This gives the Pareto frontier of the numerical solution. The same procedure is run for the set of Aimsun performance metrics. In other words, a Pareto front is found also for the Aimsun results for energy consumption, travel time, idling time and number of stops. The two Pareto fronts are then mapped onto the $\lambda_{1}-\lambda_{2}$ plane and only the intersection of the two Pareto-efficient sets is considered. The idea is to reduce the set of weights options to the ones that are Pareto efficient both for the theoretical optimization problem and for the actual traffic performance obtained in the microscopic simulator. Within this reduced set, we have chosen the weights that achieve the lowest energy consumption: $\lambda_{1}=0.4, \lambda_{2}=0.4$.

The results for both theoretical bandwidth and Aimsun performance metrics are reported in Table III. The overall network travel time is reduced by $1 \%$, although the speed 

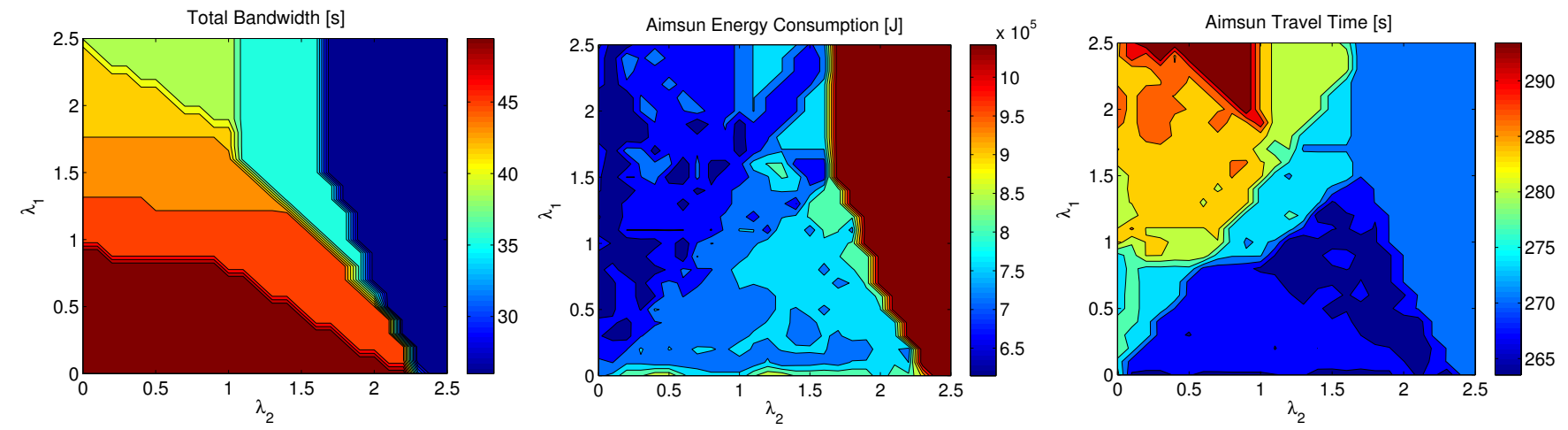

Fig. 3: Contour plots of numerical and Aimsun results. For the theoretical bandwidth, higher values (towards red) are better. For the energy consumption and the travel time lower values (towards blue) are better.

TABLE III: Numerical and Aimsun results comparison

\begin{tabular}{|l|l|c|c|c|c|}
\hline & Numerical & \multicolumn{4}{|c|}{ AIMSUN } \\
\hline & $\begin{array}{l}\text { Theoretical } \\
\text { Bandwidth }\end{array}$ & $\begin{array}{c}\text { Travel } \\
\text { Time }\end{array}$ & $\begin{array}{c}\text { Idle } \\
\text { Time }\end{array}$ & Stops & Energy \\
\hline Offset & $26 \mathrm{~s}$ & $270.3 \mathrm{~s}$ & $26.9 \mathrm{~s}$ & 3.5 & $1.09 \mathrm{E} 6 \mathrm{~J}$ \\
\hline Offset+VSL & $51 \mathrm{~s}$ & $268.3 \mathrm{~s}$ & $1.9 \mathrm{~s}$ & 0.8 & $7.03 \mathrm{E} 5 \mathrm{~J}$ \\
\hline
\end{tabular}

limit is globally smaller, as shown in Table IV. The idling time at the intersections is almost completely eliminated, and the overall energy consumption is reduced by $35.5 \%$.

TABLE IV: Optimized speed limits

\begin{tabular}{|c|c|c|}
\hline & Outbound & Inbound \\
\hline Offset+VSL control & {$[50,26,29,34,43] \mathrm{km} / \mathrm{h}$} & {$[44,44,50,50,50] \mathrm{km} / \mathrm{h}$} \\
\hline
\end{tabular}

\section{CONCLUSIONS}

This work has outlined a solution of the arterial bandwidth maximization by using both offset control and variable speed limits, and by taking into account the effects on energy consumption and network travel time. An extensive evaluation of the proposed solution has been carried out in a microscopic traffic simulator in order to assess the correlation between theoretical bandwidth and traffic performance metrics. It has been shown that the Pareto-efficient selection of weights results in a dramatic reduction of energy consumption without affecting the travel time.

Future research will include investigations into the effects of different levels of demand, including infeasible demands, on traffic performance. Also, the extension to more complex networks with cross streets and incoming flows within the arterial might open interesting analyses of the benefits of the variable speed limits on the traffic performance.

\section{ACKNOWLEDGMENTS}

The research leading to these results has received funding from the project "COMFORT" (an Associate Team between INRIA project-team NeCS and the Berkeley University project PATH) and the project "Inria@SiliconValley".

\section{REFERENCES}

[1] Z. Tian and T. Urbanik, "System Partition Technique to Improve Signal Coordination and Traffic Progression," Journal of Transportation Engineering, pp. 119-128, 2007.

[2] J. Morgan and J. Little, "Synchronizing Traffic Signals for Maximal Bandwidth," Operations Research, vol. 12, no. 6, pp. 896-912, 1964.

[3] J. Little, "The Synchronization of Traffic Signals by Mixed-Integer Linear Programming," Operations Research, vol. 14, no. 4, pp. 568594, 1966.

[4] J. Little, M. Kelson, and N. Gartner, "MAXBAND. A Versatile Program for Setting Signals on Arteries and Triangular Networks," Transportation Research Record, 1981.

[5] E. Chang, S. Cohen, C. Liu, N. Chaudhary, and C. Messer, "MAXBAND-86: Program for Optimizing Left-Turn Phase Sequence in Multiarterial Closed Networks," Transportation Research Record, vol. 1181, pp. 61-67, 1988.

[6] N. Gartner, S. Assmann, F. Lasaga, and D. Hou, "A Multi-Band Approach to Arterial Signal Optimization Traffic," Transportation Research Part B: Methodological, vol. 25B, no. 1, pp. 55-74, 1991.

[7] C. Stamatiadis and N. Gartner, "MULTIBAND-96: A Program for Variable-Bandwidth Progression Optimization of Multiarterial Traffic Networks," Transportation Research Record, vol. 1554, pp. 9-17, 1996

[8] N. Gartner and C. Stamatiadis, "Arterial-Based Control of Traffic Flow in Urban Grid Networks," Mathematical and Computer Modelling, vol. 35, pp. 657-671, 2002.

[9] W. Xianyu, H. Peifeng, and Y. Zhenzhou, "Link-Based Signalized Arterial Progression Optimization with Practical Travel Speed," Journal of Applied Mathematics, 2013.

[10] L. Lin, L. Tung, and H. Ku, "Synchronized Signal Control Model for Maximizing Progression along an Arterial," Journal of Transportation Engineering, vol. 136, no. 8, pp. 727-735, 2010.

[11] H. Tsay and L. Lin, "New Algorithm for Solving the Maximum Progression Bandwidth," Transportation Research Record, vol. 1194, pp. $15-30,1988$

[12] G. Gomes, "Bandwidth Maximization Using Vehicle Arrival Functions," IEEE Transactions on Intelligent Transportation Systems, vol. 16 , no. 4, pp. 1977-1988, 2015.

[13] J. Archer, N. Fotheringham, M. Symmons, and B. Corben, "The Impact of Lowering Speed Limits in Urban/Metropolitan Areas," Monash University Accident Research Center, Australia, Tech. Rep., 2008.

[14] S. Boyd and L. Vandenberghe, "Convex Optimization," Cambridge University Press, 2004.

[15] G. De Nunzio, C. Canudas de Wit, and P. Moulin, "Urban Traffic EcoDriving : A Macroscopic Steady-State Analysis," in IEEE European Control Conference, 2014.

[16] N. Petit and A. Sciarretta, "Optimal Drive of Electric Vehicles Using an Inversion-Based Trajectory Generation Approach," in IFAC World Congress, 2011.

[17] G. De Nunzio, C. Canudas De Wit, P. Moulin, and D. Di Domenico, "Eco-Driving in Urban Traffic Networks Using Traffic Signal Information," in IEEE Conference on Decision and Control, 2013. 